\title{
Minor QTLs mining through the combination of GWAS and machine learning feature selection
}

Wei Zhou ${ }^{1}$, Emily S. Bellis ${ }^{2}$, Jonathan Stubblefield ${ }^{1}$, Jason Causey ${ }^{1}$, Jake Qualls $^{1}$, Karl Walker $^{3}$, Xiuzhen Huang $^{1} *$

1. Department of Computer Science and Molecular Bioscience Program, Arkansas State University, Jonesboro, Arkansas, 72401

2. Department of Biology, The Pennsylvania State University, University Park, Pennsylvania, 16802

3. Department of Computer Science and Mathematics, University of Arkansas at Pine Bluff, Pine Bluff, Arkansas 71601

Wei Zhou, Department of Computer Science, Arkansas State University, Jonesboro Arkansas, 72401, Email: wzhou@astate.edu

Emily S. Bellis, Department of Biology, The Pennsylvania State University, University Park, Pennsylvania, 16802, Email: $\underline{\text { ezb336@psu.edu }}$

Jonathan Stubblefield, Department of Computer Science and MBS Program, Arkansas State University, Jonesboro, Arkansas, 72401, Email: jstubblefield@astate.edu

Jason Causey, Department of Computer Science, Arkansas State University, Jonesboro, Arkansas, 72401, Email: jcausey@astate.edu

Jake Qualls, Department of Computer Science, Arkansas State University, Jonesboro, Arkansas, 72401, Email: jqualls@astate.edu

Karl Walker, Department of Computer Science and Mathematics, University of Arkansas at Pine Bluff, 1200 North University Drive, Pine Bluff, AR 71601, Email: walkerk@uapb.edu

*Corresponding author

Dr. Xiuzhen Huang

Department of Computer Science and Molecular Bioscience Program, Arkansas State University, Jonesboro, Arkansas, 72401

Email: xhuang@astate.edu

Telephone: +1-870-680-8116 
Abstract

2 Introduction: Minor QTLs mining has a very important role in genomic selection, pathway analysis and

3 trait development in agricultural and biological research. Since most individual loci contribute little to

4 complex trait variations, it remains a challenge for traditional statistical methods to identify minor QTLs

5 with subtle phenotypic effects. Here we applied a new framework which combined the GWAS analysis

6 and machine learning feature selection to explore new ways for the study of minor QTLs mining.

7 Results: We studied the soybean branching trait with the 2,137 accessions from soybean (Glycine max)

8 diversity panel, which was sequenced by 50k SNP chips with 42,080 valid SNPs. First as a baseline

9 study, we conducted the GWAS GAPIT analysis, and we found that only one SNP marker significantly

10 associated with soybean branching was identified. We then combined the GWAS analysis and feature

11 importance analysis with Random Forest score analysis and permutation analysis. Our analysis results

12 showed that there are 36,077 features (SNPs) identified by Random Forest score analysis, and 2,098

13 features (SNPs) identified by permutation analysis. In total, there are 1,770 features (SNPs) confirmed by

14 both of the Random Forest score analysis and the permutation analysis. Based on our analysis, 328

15 branching development related genes were identified. A further analysis on GO (gene ontology) term

16 enrichment were applied on these 328 genes. And the gene location and gene expression of these

17 identified genes were provided.

18 Conclusions: We find that the combined analysis with GWAS and machine learning feature selection

19 shows significant identification power for minor QTLs mining. The presented research results on minor

20 QTLs mining will help understand the biological activities that lie between genotype and phenotype in

21 terms of causal networks of interacting genes. This study will potentially contribute to effective genomic

22 selection in plant breeding and help broaden the way of molecular breeding in plants.

23 Keywords: Machine learning, Minor QTLs, GWAS, Feature selection 


\section{Introduction}

In molecular genetics research, a remaining challenge in quantitative trait studies is the efficient mapping of minor quantitative trait loci (QTLs) to identify causative genes and understand the genetic basis of variation in quantitative traits [1]. Because the subtle influence on the phenotype of minor QTLs is easily masked by epistasis [2] and gene-environment interactions [3], minor QTLs are more difficult to be detected and analyzed. Because of this, a large fraction of the genetic architecture of most complex traits is not well understood $[4,5,6]$. Currently, almost all of genes or QTLs that have been verified were major effect ones, and the minor effect QTLs were less investigated. Several different methods have been reported to identify minor QTLs, but many of these strategies have had poor success rates [7, 8, 9]. To improve the situation, some of these studies were based on expensive experimental data from large populations. For example, Baobao et al., demonstrated a method for mapping of minor effect QTLs in maize by using super high density genotyping and large recombinant inbred population [10].

QTL-mapping algorithm based on statistical machine learning methods better estimates of QTL effects, because it eliminates the optimistic bias in the predictive performance of other QTL methods. It produces narrower peaks than other methods and hence identifies QTLs with greater precision [17]. Two machine-learning algorithms (Random Forest and boosting) have been used to analyze discrete traits in a genome-wide prediction context. It was found out that Random Forest and boosting do not need an inheritance specification model and may account for non-additive effects without increasing the number of covariates in the model or the computing time [18]. This study shows some advantages in the use of machine learning methods to analyze discrete traits in genome-wide prediction. Random Forest was shown to outperform other methods in the field datasets, with better classification performance within and across datasets. Even when tested with the main QTLs for several traits in different chromosomes, Random Forest was able to identify them, but it failed to detect significant associations when the variance explained by the QTL is low [19]. 
Besides physical QTLs mapping, machine learning methods are also used on eQTL(Expression

50

51

52

53

54

55

56

57

58

quantitative trait loci) Mapping. By using combinations of methods, an approach that relies on Random

Forests and LASSO was developed and it achieved a much higher average precision at the cost of slightly

lower average sensitivity [20]. It is observed that when combined Random Forest and other modeling

techniques, it almost always performed better than their constituent methods [21, 22]. It is observed that

Random Forests map eQTL are to be validated by independent data, when compared to competing multi-

locus and legacy eQTL mapping methods [20].

Genome-wide association studies (GWAS) is considered to be a powerful approach for dissecting complex traits $[23,24,25]$ and has been widely applied for the study of many plants, such as Arabidopsis,

rice and maize $[26,27,28,29,30,31]$. In soybean, the evaluation of several specific agronomic traits, including seed protein and oil concentration [32, 33], cyst nematode resistance [34, 35], and flowering time [36] were conducted through GWAS. Plant architecture related traits (PATs) are of great importance

for soybean and many crops. Studies in past decades indicated that PATs are mainly affected by minor effect quantitative traits loci (QTLs), especially as reflected in the Nested Association Mapping (NAM) population $[37,38,39]$.

From these previous studies, however, minor QTLs are hard to be detected mainly because their contribution is subtle. It is challenging for current statistical methods to detect them. For example, most of statistic methods are based on the variance analysis, such as ANOVA, and they usually need a larger population size to detect minor QTLs.

In this study, with soybean branching as the focused trait, we combined the GWAS analysis and machine learning feature selection, to explore the application of a new analysis framework in minor QTLs mining in plants. As a result, we identified 328 minor genes and 1770 effective SNP markers related to soybean branching development. Our analysis results with the new framework for minor QTLs mining would benefit the genomic selection, the pathway analysis and organism development research.

\section{Methods:}




\section{Dataset}

75 The original genotypic data is from soybase data bank : https://soybase.org/snps/. The SoySNP50K

76 iSelect BeadChip has been used to genotype the USDA Soybean Germplasm Collection [46]. The

77 complete data set for 20,087 G. max accessions genotyped with 42,509 SNPs is available.

78 Soybean accessions and phenotypic data used in this study were obtained from the USDA Soybean

79 Germplasm Collection (http://www.ars-grin.gov/npgs/). Branching phenotype data was extracted and

80 used for analysis. Missing data and SNPs with minor allele frequencies below 0.1 were excluded, leaving

8142,080 SNPs for GWAS.

\section{Genome wide association study (GWAS)}

83 Association analysis and estimation of each SNP effect was implemented in GAPIT software (version 2)

84 [47]. The regression linear model (GLM), and the mixed linear model (MLM) methods were used as

85 described by Tang et al. [45]. Default parameters of the SUPER model were used: sangwich.top =

"MLM," sangwich.bottom = "SUPER," LD =0.1. The significant P-value cut-off was set as $\mathrm{p}=3.45 \mathrm{e}-07$,

87 equivalent to $\alpha$ level of 0.05 after Bonferroni correction. The efficient mixed-model association with

corrections for kinship and population structure was applied. Three PCs generated from GAPIT were

included as covariates. The SNPs with a minor allele frequency (MAF) higher than 0.01 were used to

91 association tests. The cutoff of significant association was a False Discovery Rate (FDR) adjusted P-value

92 less than 0.1 using the Benjamini and Hochberg procedure to control for multiple testing. Significant

93 SNPs were defined if showing a minus $\log 10$-transformed $\mathrm{P} \geq 3$. SNPs with a genetic distance less than 2

$94 \mathrm{cM}$ were considered to be in a $\mathrm{LD}$ extension block and belong to the same SNP cluster.

\section{Data preprocessing}

96 In machine learning feature selection analysis, all of nucleotides in genotype data was added the rs

97 (Reference SNP cluster ID) information and transformed as rs + nucleotide (Sup_Table7). The whole

98 dataset was divided into 11 subsets based on different $\mathrm{P}$-value levels for a further analysis in machine 
learning models. The genotype data used in regression and feature importance analysis were encoded by

100

101

102

103

104

105

106

107

108

109

110

111

113

114

115

116

117

OneHotEncoder after labelencoding.

\section{Feature importance analysis}

Feature importance analysis explains what features have the biggest impact on predictions in testing model. Permutation importance is a kind of global model-agnostic method and calculated after a model has been fitted. Compared to most other approaches, permutation importance is fast to calculate and widely used. Random forest is one of the most effective machine learning models for predictive analytics capable of performing both regression and classification tasks and able to capture non-linear interaction between the features and the target. It is very good at handling categorical features with fewer than hundreds of categories [49]. The character of permutation importance consists with the properties we would want a feature importance measure to have. In this research we applied the random regressor in permutation importance analysis and Random Forest score analysis for all of 2137 samples and 42080 features (SNPs).

\section{Gene Ontology analysis}

SNPs identified by feature importance analysis were searched in SoyBase data site (https://soybase.org/snps/) by rs number. And the flank sequence of corresponding SNP was used to BLAST in Glycine max Genome DB database (http://www.plantgdb.org/GmGDB/) for confirmation.

The gene names which SNPs hit to the same location (including CDS, UTR and intron) were collected for GO (gene ontology) analysis. All the genes identified by BLAST were analyzed by GO term enrichment tool at SoyBase website (https://soybase.org/goslimgraphic_v2/dashboard.php). The GO enrichment information, related charts and gene location map were generated by GO term enrichment tool at SoyBase website.

\section{Results:}

\section{Genome Wide Association Study (GWAS) for soybean branching}

A genome-wide association study (GWAS) of soybean branching was conducted with 42,080 SNP markers in the GAPIT (Genome Association and Prediction Integrated Tool) software using a mixed 
markers, there are 18 markers with P-value less than 0.005, 32 markers with P-value less than 0.01 and

161 makers with P-value less than 0.05(Table 1. and Sup_Table1.). Associations between phenotypes and genetic markers are displayed as Manhattan plots (Fig. 1) and (Sup_Table1). P-values were displayed in negative $\log$ scale with base of $10(-\log 10(\mathrm{P}))$ against the physical map positions of genetic markers. We set a threshold of $-\log 10(0.1 / 42080)=5.624(42080$ is the SNP marker numbers $)$ to identify SNPs significantly associated with a trait. In total of 161 which P-value is less than 0.05 , only SNP marker

132 ss715607451 were significantly $(-\log 10(\mathrm{p})=9.524328812)$ associated with soybean branching trait. annotated genes are found (Table 1), but none of them is reported as branching related.

\section{Feature importance analysis}

Please refer to Fig. 3 for a summary chart of our feature importance analysis. In the following we give the details of our analysis results.

In general, feature importance analysis is based on the understanding how the features in the

142 testing model contribute to the prediction model. Feature importance includes local model-agnostic

143 feature importance and global model-agnostic feature importance. Since local measures focus on the 144 contribution of features for a specific prediction, whereas global measures take all predictions into 145 account. Here we applied permutation feature importance, a global model-agnostic approach, with the 146 Random Forest algorithm as the core. After evaluating the performance of the models, we permuted the 147 values of a feature of interest and re-evaluate the model performance. The average reduction in impurity 148 across all trees in the forest due to each feature was computed. 

them, 971 features (SNPs) have weights bigger than 1E-06, 952 features (SNPs) in total have weights bigger than 1E-05 and 872 features (SNPs) have weights bigger than 0.0001(Sup_Table2.). Our results also showed that there are 1124 features in total with negative weight values. Among them, 1107 features (SNPs) have weights smaller than -1E-05, 939 features (SNPs) have weights smaller than 1E-04 (Sup_Table2.). There are 39982 features with weight zero in the Random Forest regression model, and these features account for around $95.014 \%$ of the total number of features (SNPs) (Sup_Table2.). Table 2 showed the top 20 features with higher importance in both the positive side and negative side. showed that there are 36077 features in total got a score bigger than 1E-07. Among them, 33121 features

\section{Comparison of different methods for feature importance analysis} (SNPs) with P-value < 1 (69 positives and 77 negatives) (Fig. 2, Sup_Table4.). 
175

176

177

178

179

180

data subsets from the GAPIT analysis, the accurate level close to the data set those with P-value $<1$. For SNPs with P-value less than 1 in the GAPIT analysis, the RMSE value was 0.2601 and the $\mathrm{R}^{2}$ value was 0.7810, but there were 3451 features (SNPs) applied (Table 2). In other words, our results showed that 1770 features (SNPs) from feature selection could reach the same accuracy as the 3451 features (SNPs) with P-value less than 1.0. The analysis showed that feature importance analysis could help lower the feature size and increase the computation efficiency.

Based on the above analysis, we searched all 1170 SNPs which were confirmed by both of Random Forest score and Permutation analysis in soybean genome. We found that 328 SNPs hit the annotated genes (Sup_Table4). To identify biological processes these 328 genes participate in, we further applied the GO (gene ontology) term enrichment analysis for all of them. Our result showed that the functional group for biological process, cellular component and molecular function were highly enriched by most of these 328 genes (Fig. 3, 4, and 5, Sup_Table5). In biological process, 66 genes (times) were classified into 16 GO term classes and 14 genes had no specific GO term to assign (Fig. 3, Sup_Table5). In cellular component class, 388 genes (times) were classified into 18 GO classes and 14 genes had no specific GO term to assign (Fig. 4, Sup_Table5). In molecular function class, 264 genes (times) were classified into 17 GO classes and 14 genes had no specific GO term to assign (Fig. 5, Sup_Table5). As is common with GO analysis, some genes were classified differently under different GO terms (Sup_Table5).

Gene location mapping results showed that all of these 328 genes are scattered on chromosome 1 to chromosome 18. There were no branching related genes located in chromosome 19 and chromosome 20 (Fig. 6). The inquiry term "branching" was searched in Soybase and 35 genes were found (Sup_Table4). To make a comparison, the location of these 35 genes were also marked on Fig. 6. The gene expression information of all 328 genes identified in this research were searched against Soybase for a further analysis (Sup_Table 6).

\section{Discussion:}

\section{Minor QTLs and genomic selection}



breeding population, in which whole genome SNPs (single-nucleotide polymorphisms) markers can be used to predict breeding values. Genomic selection has been proved to increase breeding efficiency in both plant and animal breeding, such as dairy cattle, pig, rice and soybean [41]. To get an accurate prediction in genomic selection, we need a better understanding of the population of SNP makers and the contribution of each markers. In the last decade, efforts of global international collaborations have

207 revealed numerous loci that influence traits development in different organism by genotyping and phenotyping very large cohorts of individuals. However, the effects of single alleles explain only a small 209 portion of the heritable variability [42]. Although some traits loci are found, these loci alone do not point to the underlying mechanism responsible for the association, which is due to complex gene interactions in biological activities. To identify genes and pathways responsible for variation in quantitative traits, it is still a central challenge of modern genetics.

214 more importance in molecular breeding and commercial breeding since the enrichment of minor alleles

215 can enhance the control accuracy of phenotype performance [43]. In this research, we applied a new

216 framework which combined the GWAS analysis and different feature selection methods to explore minor

217 QTLs/alleles and their importance in soybean branching. Compare to the P-value method in GWAS 218 analysis, the feature importance analysis we used in this research explored 36077 features in total with a 219 score higher than 1E-07, which is about ten times as the number of the features identified in GWAS 220 analysis with P-value less than 1.0. Based on the Permutation feature importance analysis, we explored 221974 features with positive effects on soybean branching development and 1124 features with negative 222 effects on soybean branching development (Table 2 and Sup_Table2). Either in linkage mapping or in 223 association mapping, it is difficult to find the QTLs which have negative contribution to a trait, even we

224 all know there are negative QTLs/alleles involved in all biological activities. From our analysis and 225 testing results, the new framework we used in this research is superior to the traditional P-value based 226 methods in molecular genetics analysis. Actually, in GWAS analysis, there is only one SNP 

none of them is reported as branching related. All of these information are very important to genomic selection and could lead to an accurate prediction in genomic selection a further study in future.

\section{Feature importance analysis and its applications}

In this research, we applied three kind of feature importance analysis, permuted feature importance, feature importance scoring and P-value analysis through GAPIT. We employed the Random

234 Forest regression algorithm in permuted feature importance and feature importance scoring analysis. It is 235 reported that the feature importance based methods are applicable if we are going to use a tree-based 236 model for making predictions [44]. Random Forest is one of the most effective machine learning models 237 for predictive analytics capable of performing both regression and classification tasks and able to capture non-linear interaction between the features and the target [45]. In random Forest, features that tend to split nodes closer to the root of a tree will result in a larger importance value. Node splits based on this feature on average result in a large decrease of node impurity. Permutation feature importance is a modelagnostic approach and is calculated after a model has been fitted. The values of a feature of interest and reevaluate model performance is permutated after evaluating the performance of model. The observed mean decrease in performance indicates feature importance. The performance decrease can be compared

244 on the test set as well as the training set. Only the latter will tell us something about generalizable feature 245 importance.

As we mentioned above, one of the biggest problems facing GWAS analysis is difficult to detect

247 quantitative traits which controlled by multiple genes, Association mapping and bi-parent mapping good

248 for major QTLs but not minor QTLs, Minor QTLs are important for quantitative traits but hard to be 249 detected by traditional genetic research, Machine learning methods open a door for minor QTLs mining,

250 special for non-model organisms with less research basis. Our results showed that the new framework 251 displays much powerful ability in minor QTLs mining than conventional analysis methods. We can 
252 expect many discoveries will be made through applications of different machine learning methods to

253 genomics data, particularly in genomic selection research.

Conclusions:

Accurate prediction of genomic breeding values is a central challenge to contemporary plant and

256 animal breeders. Minor QTLs play very important roles in this procedure, but we know little about the

257 minor QTLs in most traits' development. To understand how many genes and which genes involved in

258 the trait's development is the prerequisites of breeding prediction. In this research, we combined the

259 GWAS analysis and feature selection with machine learning methods, and explored the new framework in

260 minor QTLs mining. The framework provides a way for finding minor QTLs and better estimates of the

261 QTL effects supportable by the data. Unlike QTL mapping through linkage mapping, this framework does

262 not require a genetic map. It is therefore applicable to any species or population. This research on minor

263 QTLs miming will contribute to trait's development and gene pathway analysis in further studies. 


\section{Abbreviations}

267 SNPs: single-nucleotide polymorphisms

268 GWAS: genome-wide association study

269 CDS: coding region sequence

270 UTR: untranslated region

271 GO: gene ontology

272 BLAST: Basic Local Alignment Search Tool

273 


\section{Declarations}

275

276

277 discussions on this research.

278 Funding

279 This work was partially supported by National Institute of Health NCI grant U01CA187013, and National

280 Science Foundation with grant number 1452211, 1553680, and 1723529, National Institute of Health

281 grant R01LM012601, as well as partially supported by National Institute of Health grant from the

282 National Institute of General Medical Sciences (P20GM103429).

283 Availability of data and materials

The original dataset is publically available. And our intermediate analysis results and code used for analysis with this study are available from the corresponding author upon request. relationships that could be construed as a potential conflict of interest.

\section{Authors' contributions}

WZ participated in the statistical analyses, data processing and writing the manuscript. XH participated in

291 conceiving the presented idea, development of the software, discussions of the results, and drafted the

292 manuscript. EB, JS, JC, JQ and KW collaborated with statistical analyses, data processing, interpretation,

293 data analysis support, and writing of the manuscript. All the authors approved the manuscript.

\section{Ethics approval and consent to participate}

$295 \quad$ NA

\section{Consent for publication}

297 NA

\section{Competing interests}

299 The authors declare that they have no competing interests. 


\section{References}

1. Mackay TF, Stone EA, Ayroles JF. The genetics of quantitative traits: challenges and prospects. Nature Reviews Genetics. 2009; 10:565.

2. Carlborg O, Haley CS. Epistasis: too often neglected in complex trait studies? Nature reviews Genetics 2004; 5: 618-25.

3. Smith EN, Kruglyak L. Gene-environment interaction in yeast gene expression. PLoS biology. 2008;

$306 \quad 6: \mathrm{e} 83$.

307 4. Mackay T. F. The genetic architecture of quantitative traits. Annu. Rev. Genet. 2001; 35: 303-339.

5. Allen HL, Estrada K, Lettre G, Berndt SI, Weedon MN, Rivadeneira F, Willer CJ, Jackson AU,

Vedantam S, Raychaudhuri S, Ferreira T. Hundreds of variants clustered in genomic loci and biological pathways affect human height. Nature. 2010; 467: 832-838.

313 using common SNPs. Nature genetics. 2011; 43: 519-525.

314 7.Flint J, Valdar W, Shifman S, Mott R. Strategies for mapping and cloning quantitative trait genes in 315 rodents. Nature Reviews Genetics. 2005; 6: 271-286.

316 8. Darvasi A. Experimental strategies for the genetic dissection of complex traits in animal models.

317 Nature genetics. 1998; 18: 19-24.

318 9. Satagopan JM, Sen S, Churchill GA. Sequential quantitative trait locus mapping in experimental 319 crosses. Statistical applications in genetics and molecular biology. 2007;6(1).

320 10. Wang B, Liu H, Liu Z, Dong X, Guo J, Li W, Chen J, Gao C, Zhu Y, Zheng X, Chen Z. Identification 321 of minor effect QTLs for plant architecture related traits using super high density genotyping and large 322 recombinant inbred population in maize (Zea mays). BMC plant biology. 2018;18:17.

323 11. Ratner B. Statistical and machine-learning data mining: Techniques for better predictive modeling and 324 analysis of big data. Chapman and Hall/CRC; 2017 Jul 12. 
12. Libbrecht MW, Noble WS. Machine learning applications in genetics and genomics. Nature Reviews Genetics. 2015; 16:321.

13. Berman JJ. Principles of big data: preparing, sharing, and analyzing complex information. Newnes; 2013.

14. Bassel GW, Glaab E, Marquez J, Holdsworth MJ, Bacardit J. Functional network construction in Arabidopsis using rule-based machine learning on large-scale data sets. The Plant Cell. 2011; 23:3101-16. functional, transcriptional, physical interaction, and metabolic networks. The Plant Cell. 2012; 24:3859-

16. Long N, Gianola D, Rosa GJ, Weigel KA, Avendano S. Machine learning classification procedure for selecting SNPs in genomic selection: application to early mortality in broilers. Journal of animal breeding and genetics. 2007; 124:377-89. trait loci by machine learning. BMC genetics. 2008; 9:35. machine learning. Genetics Selection Evolution. 2011; 43:7.

341 19. Minozzi G, Pedretti A, Biffani S, Nicolazzi EL, Stella A. Genome wide association analysis of the

342 16th QTL-MAS Workshop dataset using the Random Forest machine learning approach. InBMC

343 proceedings 2014 Oct (Vol. 8, No. 5, p. S4). BioMed Central.

344 20. Michaelson JJ, Alberts R, Schughart K, Beyer A. Data-driven assessment of eQTL mapping methods. 345 BMC genomics. 2010; 11:502.

346 21. Hastie T, Tibshirani R, Friedman JH. The elements of statistical learning: data mining, inference, and 347 prediction. New York: Springer: 2009.

348 22. Ackermann M, Clément-Ziza M, Michaelson JJ, Beyer A. Teamwork: improved eQTL mapping using 349 combinations of machine learning methods. PloS one. 2012: 24:1-8 
23. Korte A, Farlow A. The advantages and limitations of trait analysis with GWAS: a review. Plant Methods. 2013; 9:29-37. Association for Plant Height and Flowering Time across 15 tropical maize populations under managed drought stress and well-watered conditions in sub-Saharan Africa. Crop Sci. 2016; 56(5):2365-2378. association study for agronomic traits in soybean using SNP markers and SNP based haplotype analysis.

357 PLoS One. 2017; 12(2).

358 26. Atwell S, Huang YS, Vilhjalmsson BJ, Willems G, Horton M, Li Y, et al. Genome-wide association 359 study of 107 phenotypes in Arabidopsis thaliana inbred lines. Nature. 2010; 465:627-31.

360 27. Huang X, Wei X, Sang T, Zhao Q, Feng Q, Zhao Y, et al. Genome-wide association studies of 14 agronomic traits in rice landraces. Nat Genet. 2010; 42:961-7. time and grain yield traits in a worldwide collection of rice germplasm. Nat Genet. 2012; 44:32-9.

364 29. Chen W, Gao Y, Xie W, Gong L, Lu K, Wang W, et al. Genome-wide association analyses provide genetic and biochemical insights into natural variation in rice metabolism. Nat Genet. 2014; 46:714-21.

367 architecture of maize flowering time. Science. 2009; 325:714-8.

368 31. Li H, Peng Z, Yang X, Wang W, Fu J, Wang J, et al. Genome-wide association study dissects the 369 genetic architecture of oil biosynthesis in maize kernels. Nat Genet. 2013; 45:43-50.

370 32. Hwang EY, Song Q, Jia G, Specht JE, Hyten DL, Costa J, et al. A genome-wide association study of 371 seed protein and oil content in soybean. BMC Genomics. 2014; 15:1-12.

372 33. Bandillo N, Jarquin D, Song QJ, Nelson R, Cregan P, Specht J, et al. A population structure and 373 genome-wide association analysis on the USDA soybean germplasm collection. Plant Genome. 2015; $3748: 1-13$. 
34. Han Y, Zhao X, Cao G, Wang Y, Li Y, Liu D, et al. Genetic characteristics of soybean resistance to HG type 0 and HG type 1.2.3.5.7 of the cyst nematode analyzed by genome-wide association mapping. BMC Genomics. 2015; 16:598-608.

35. Vuong TD, Sonah H, Meinhardt CG, Deshmukh R, Kadam S, Nelson RL, et al. Genetic architecture of cyst nematode resistance revealed by genome-wide association study in soybean. BMC Genomics. $2015 ; 16: 593-605$.

36. Zhang J, Song Q, Cregan PB, Nelson RL, Wang X, Wu J, et al. Genome-wide association study for flowering time, maturity dates and plant height in early maturing soybean (Glycine max) germplasm. BMC Genomics. 2015; 16:217-27.

37. Brown PJ, Upadyayula N, Mahone GS, Tian F, Bradbury PJ, Myles S, Holland JB, Flint-Garcia S, McMullen MD, Buckler ES, Rocheford TR. Distinct genetic architectures for male and female inflorescence traits of maize. PLoS genetics. 2011; 7(11).

38. Tian F, Bradbury PJ, Brown PJ, Hung H, Sun Q, Flint-Garcia S, Rocheford TR, McMullen MD, Holland JB, Buckler ES. Genome-wide association study of leaf architecture in the maize nested association mapping population. Nat Genet. 2011; 43(2):159-162.

39. Peiffer JA, Romay MC, Gore MA, Flint-Garcia SA, Zhang Z, Millard MJ, Gardner CAC, McMullen MD, Holland JB, Bradbury PJ, et al. The genetic architecture of maize height. Genetics. 2014; 196(4):1337-1356.

40. Zou H, Hastie T. Regularization and variable selection via the elastic net. Journal of the royal statistical society: series B (statistical methodology). 2005; 67:301-20.

41. Shamshad M, Sharma A. The Usage of Genomic Selection Strategy in Plant Breeding. InNext Generation Plant Breeding 2018 Nov 5. IntechOpen.

42. Manolio TA, Collins FS, Cox NJ, Goldstein DB, Hindorff LA, Hunter DJ, McCarthy MI, Ramos EM, Cardon LR, Chakravarti A, Cho JH. Finding the missing heritability of complex diseases. Nature. 2009; 461: 747-753. 
401 Quail MA, Moses A. Revealing the genetic structure of a trait by sequencing a population under selection.

402 Genome research. 2011; 21(7):1131-8.

403 44. Cao DS, Xu QS, Liang YZ, Chen X, Li HD. Automatic feature subset selection for decision tree-

404 based ensemble methods in the prediction of bioactivity. Chemometrics and Intelligent Laboratory

405 Systems. 2010; 103:129-36.

406 45. Voyant C, Notton G, Kalogirou S, Nivet ML, Paoli C, Motte F, Fouilloy A. Machine learning

407 methods for solar radiation forecasting: A review. Renewable Energy. 2017 May 1;105:569-82.

408 46. Song Q, Hyten DL, Jia G, Quigley CV, Fickus EW, Nelson RL, Cregan PB. Fingerprinting soybean

409 germplasm and its utility in genomic research. G3: Genes, Genomes, Genetics. 2015 Oct 1;5(10):1999-

4102006.

411 47. Tang Y, Liu X, Wang J, Li M, Wang Q, Tian F, Su Z, Pan Y, Liu D, Lipka AE, Buckler ES. GAPIT

412 version 2: an enhanced integrated tool for genomic association and prediction. The plant genome. 2016;

$4139(2)$.

414 48. Zou H, Hastie T. Regression shrinkage and selection via the elastic net, with applications to

415 microarrays. JR Stat Soc Ser B. 2003; 67:301-20.

416 49. Zhou L, Pan S, Wang J, Vasilakos AV. Machine learning on big data: Opportunities and challenges.

417 Neurocomputing. 2017; 237:350-61. 


\section{Tables and table legends}

Table 1. Summary of SNP markers with P-value less than 0.005 from GWAS analysis

\begin{tabular}{|c|c|c|c|c|c|c|}
\hline SNP & Location & Chr.* & Position & P.value & $\mathbf{R M S}^{* *}$ & value \\
\hline ss715607451 & intergenic & 10 & 45054553 & $2.99 \mathrm{E}-10$ & 0.363021 & 9.524329 \\
\hline ss715632223 & intergenic & 18 & 55622046 & 2.32E-05 & 0.356302 & 4.634512 \\
\hline ss715613636 & intergenic & 12 & 8904870 & 2.79E-05 & 0.356195 & 4.554396 \\
\hline ss715579744 & Glyma01g35330 & 1 & 48727937 & 0.000209 & 0.355028 & 3.679931 \\
\hline ss715622025 & intergenic & 15 & 46324641 & 0.000363 & 0.35471 & 3.439907 \\
\hline ss715622023 & intergenic & 15 & 46299552 & 0.000531 & 0.354493 & 3.275199 \\
\hline ss715579749 & Glyma01g35370 & 1 & 48751448 & 0.000605 & 0.354419 & 3.218473 \\
\hline ss715613329 & intergenic & 12 & 6846229 & 0.000809 & 0.354254 & 3.092144 \\
\hline ss715579747 & intergenic & 1 & 48741524 & 0.000883 & 0.354204 & 3.054065 \\
\hline ss715637835 & intergenic & 20 & 37318170 & 0.001282 & 0.353993 & 2.892276 \\
\hline ss715607752 & Glyma10g39840 & 10 & 48017555 & 0.001568 & 0.353879 & 2.804728 \\
\hline ss715613193 & intergenic & 12 & 5623543 & 0.002378 & 0.353645 & 2.623833 \\
\hline ss715638884 & Glyma20g38610 & 20 & 47292145 & 0.002454 & 0.353628 & 2.610076 \\
\hline ss715638808 & Glyma20g37960 & 20 & 46826697 & 0.003846 & 0.353377 & 2.414971 \\
\hline ss715590469 & intergenic & 5 & 30305516 & 0.003889 & 0.353371 & 2.410177 \\
\hline ss715633540 & intergenic & 19 & 25950203 & 0.004234 & 0.353324 & 2.373279 \\
\hline ss715583971 & intergenic & 2 & 8220222 & 0.004556 & 0.353283 & 2.341369 \\
\hline
\end{tabular}

*Chr. indicates chromosome number.

**RMS indicates R-square of Model with SNP

Location indicates the SNPs in or out of an annotated gene. 
Table 2. Top 20 features with higher importance from permutation analysis

\begin{tabular}{|c|c|c|c|c|c|c|c|c|c|}
\hline \multirow[b]{2}{*}{ rs\# } & \multirow[b]{2}{*}{ weight } & \multicolumn{2}{|c|}{ positive weight } & \multirow[b]{2}{*}{ score } & \multirow[b]{2}{*}{ rs\# } & \multicolumn{2}{|c|}{ negative weight } & \multirow[b]{2}{*}{ P-value } & \multirow[b]{2}{*}{ score } \\
\hline & & Std** & P-value & & & weight & Std $* *$ & & \\
\hline ss715636302 & 0.006481 & 0.000887 & 1 & 0 & ss715584181 & -0.01309 & 0.006558 & 1 & $5.19 \mathrm{E}-05$ \\
\hline ss715632046 & 0.006242 & 0.000954 & 1 & 0 & ss715606461 & -0.01298 & 0.003566 & 1 & $1.08 \mathrm{E}-05$ \\
\hline ss715586408 & 0.005429 & 0.001522 & 1 & $4.26 \mathrm{E}-05$ & ss715611174 & -0.01231 & 0.008024 & 1 & $7.61 \mathrm{E}-06$ \\
\hline ss715629729 & 0.005398 & 0.00163 & 1 & $3.52 \mathrm{E}-07$ & ss715601294 & -0.01182 & 0.002501 & 1 & $1.57 \mathrm{E}-05$ \\
\hline ss715639086 & 0.005334 & 0.001063 & 1 & 0 & ss715621258 & -0.01084 & 0.006296 & 1 & $2.42 \mathrm{E}-06$ \\
\hline ss715600775 & 0.0051 & 0.001249 & 1 & $1.62 \mathrm{E}-05$ & ss715636617 & -0.01053 & 0.00258 & 1 & 0 \\
\hline ss715634776 & 0.004993 & 0.000713 & 1 & 0 & ss715614457 & -0.01017 & 0.004173 & 1 & $5.59 \mathrm{E}-06$ \\
\hline ss715621250 & 0.004987 & 0.002158 & 1 & $2.42 \mathrm{E}-06$ & ss715584279 & -0.00995 & 0.002648 & 1 & $5.13 \mathrm{E}-05$ \\
\hline ss715606657 & 0.004812 & 0.002458 & 0.499356 & $1.06 \mathrm{E}-05$ & ss715616125 & -0.00898 & 0.001576 & 1 & $4.58 \mathrm{E}-06$ \\
\hline ss715610241 & 0.004781 & 0.001113 & 1 & $8.27 \mathrm{E}-06$ & ss715638986 & -0.00891 & 0.007572 & 1 & 0 \\
\hline ss715597582 & 0.004612 & 0.0013 & 1 & $1.97 \mathrm{E}-05$ & ss715619437 & -0.00862 & 0.002983 & 1 & $3.08 \mathrm{E}-06$ \\
\hline ss715611890 & 0.004579 & 7.37E-05 & 1 & $7.16 \mathrm{E}-06$ & ss715610413 & -0.00722 & 0.002548 & 0.263147 & $8.13 \mathrm{E}-06$ \\
\hline ss715632589 & 0.004394 & 0.004083 & 1 & 0 & ss715604675 & -0.00668 & 0.00313 & 1 & $1.23 \mathrm{E}-05$ \\
\hline ss715580947 & 0.004045 & 0.0068 & 1 & $8.9 \mathrm{E}-05$ & ss715605467 & -0.00666 & 0.00415 & 1 & $1.17 \mathrm{E}-05$ \\
\hline ss715596598 & 0.003836 & 0.003571 & 1 & $2.09 \mathrm{E}-05$ & ss715607569 & -0.00632 & 0.006535 & 1 & $9.93 \mathrm{E}-06$ \\
\hline ss715617936 & 0.003701 & 0.002292 & 0.846353 & $3.72 \mathrm{E}-06$ & ss715588364 & -0.00615 & 0.001517 & 1 & $3.63 \mathrm{E}-05$ \\
\hline ss715579007 & 0.003535 & 0.001199 & 1 & 0.00025 & ss715590471 & -0.00558 & 0.001448 & 1 & $3.1 \mathrm{E}-05$ \\
\hline ss715609664 & 0.003281 & 0.001464 & 1 & $8.63 \mathrm{E}-06$ & ss715598227 & -0.00557 & 0.005702 & 1 & $1.89 \mathrm{E}-05$ \\
\hline ss715625697 & 0.003213 & 0.000729 & 1 & $1.09 \mathrm{E}-06$ & ss715632123 & -0.00539 & 0.0025 & 1 & 0 \\
\hline ss715586600 & 0.003188 & 0.000131 & 1 & $4.18 \mathrm{E}-05$ & ss715607399 & -0.0051 & 0.001592 & 1 & $1.01 \mathrm{E}-05$ \\
\hline
\end{tabular}

This table shows the top 20 features with higher importance in both the positive side and negative side from permutation analysis.

*rs\# refers to Reference SNP cluster ID

Weight indicates the feature importance weight of SNP by permutation analysis

** std refers to Standard Deviation

Score indicates the score of each feature by Random Forest score analysis

$\mathrm{P}$-value is calculated by the GAPIT software 
Figures and Figure legends

Fig. 1. Manhattan plots of genome-wide association studies (GWAS) for soybean branching

\section{MLM.Branching}

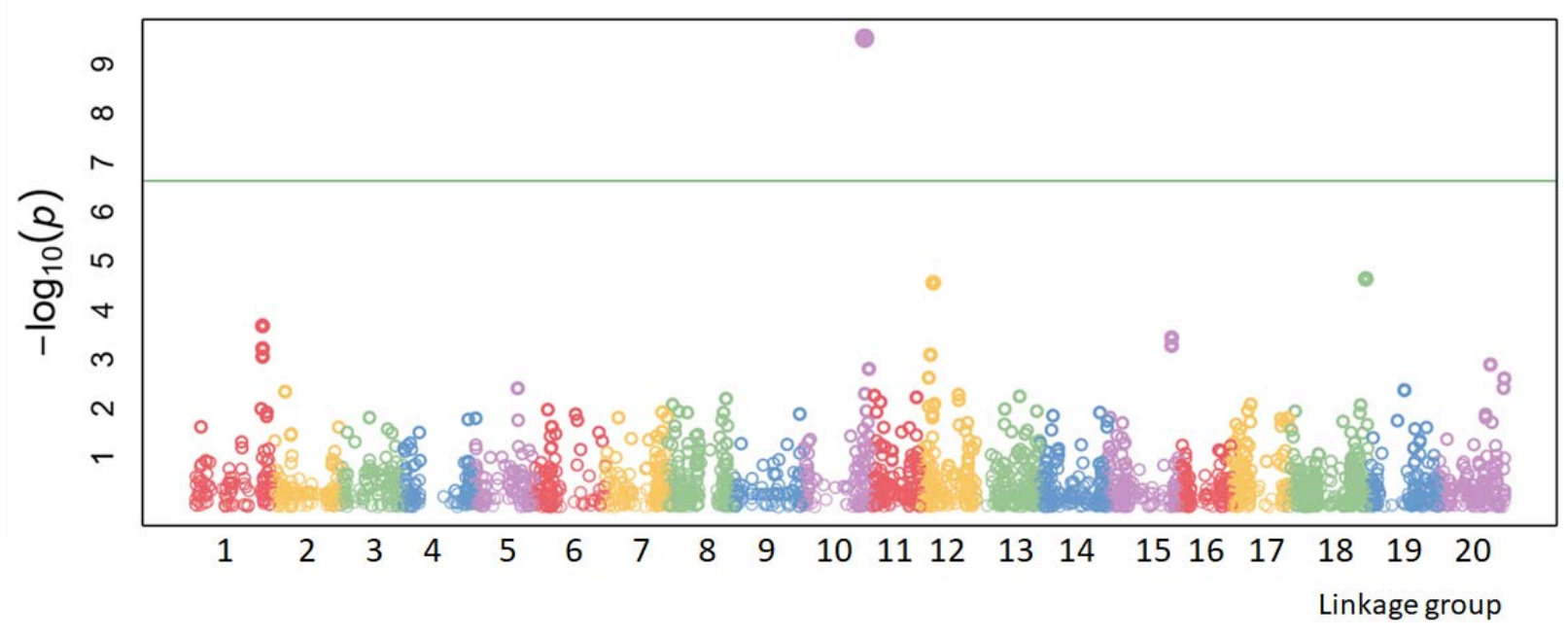

Manhattan plots of genome-wide association studies (GWAS) for soybean branching measured with the mixed linear model (MLM). The X-axis is the genomic position of the SNPs in each linkage group, and the Y-axis is the negative log base 10 of the P-values. Each chromosome is colored differently. SNPs with stronger associations with the trait will have a larger Y-coordinate value. The general and highly significant trait-associated SNPs are distinguished by the green threshold lines. Genetic markers are positioned by their chromosomes and ordered by their base-pair positions. Genetic markers on adjacent chromosomes are displayed with different colors. The strength of the association signal is displayed in two ways. One indicator of strength is the height on the vertical axis for-log P-values; the greater the height, the stronger the association. The other indicator is the degree of filling in the dots; the greater the area filled within the dot, the stronger the association. 
Fig. 2. The summary chart of feature importance analysis

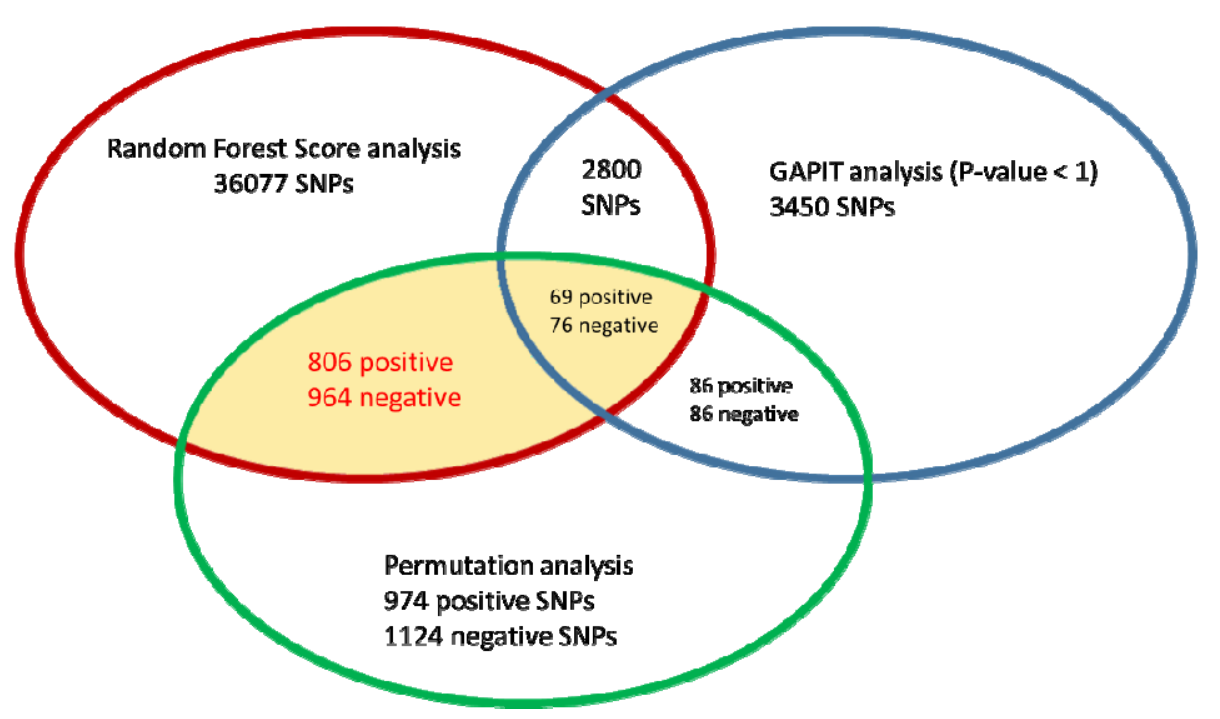

This shows a summary of feature importance analysis by the different methods. Blue circle refers to the SNPs with P-value less than 1 identified by GAPIT software; and a total of 3450 SNPs were identified. Green circle refers to the 974 SNPs with positive weight and 1124 SNPs with negative weight, identified by permutation importance analysis. Red circle refers the 36077 SNPs, identified by Random Forest score (score $>=1 \mathrm{E}-04$ ). The numbers inside the intersection refers to the SNPs, confirmed by both methods. 2800 SNPs were identified by Random Forest score analysis, with P-value less than 1 . A total of 1770 SNPs (with 806 SNPs with positive weight and 964 SNPs with negative weight) were confirmed by both of Random Forest score analysis and permutation analysis (highlighted in yellow). 86 SNPs with positive weight and 86 SNPs with negative weight were identified by permutation analysis, with P-value less than 1. The 69 SNPs with positive weight and 76 SNPs with negative weight were confirmed by both of Random Forest score analysis and permutation analysis, with P-value less than 1(highlighted in yellow). 
Fig. 3. Biological Process Classification

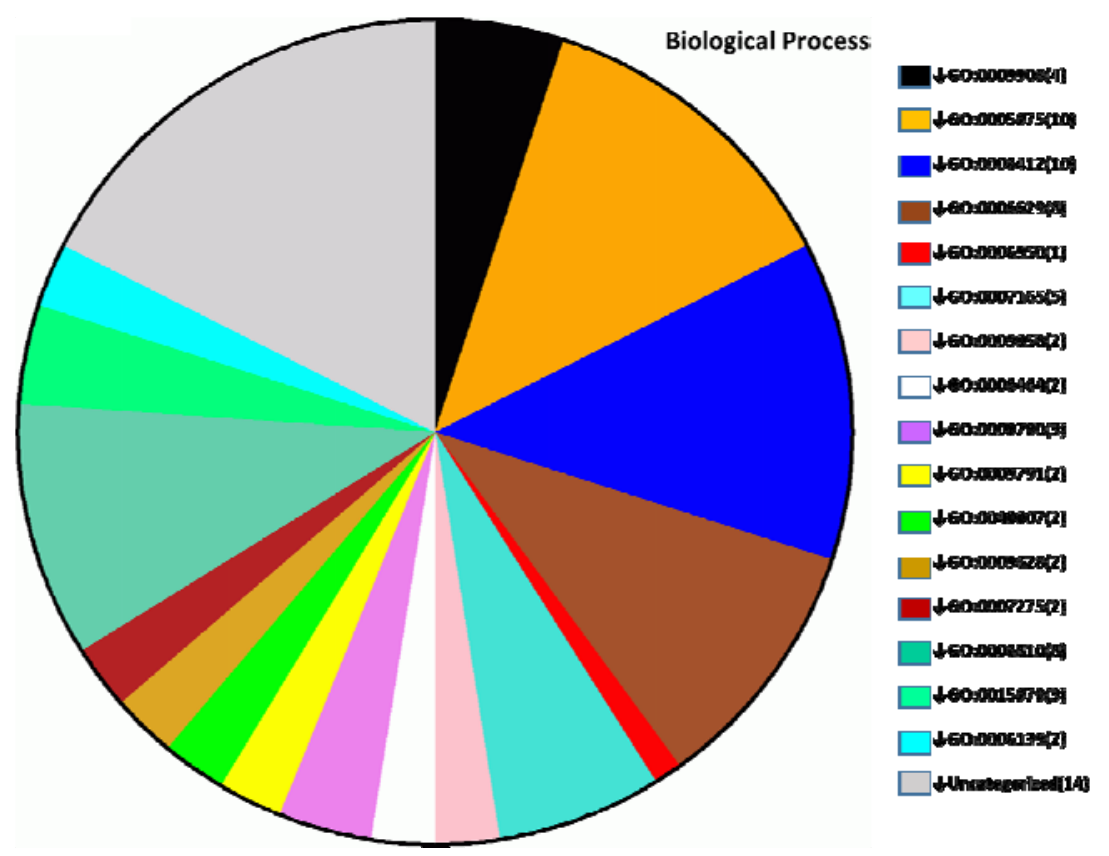

This shows the biological process classification based on GO enrichment analysis. There are 66 genes were classified into 16 GO classes, they are GO:0009908 (Flower Development), GO:0005975(Carbohydrate Metabolic Process), GO:0006412(Translation), GO:0006629 (Lipid Metabolic Process), GO:0006950(Response To Stress), GO:0007165(Signal Transduction), GO:0009058(Biosynthetic Process), GO:0006464 (Protein Modification Process), GO:0009790 (Embryo Development), GO:0009791 (Post-embryonic Development), GO:0040007 (Growth), GO:0009628 (Response To Abiotic Stimulus), GO:0007275 (Multicellular Organismal Development), GO:0006810(Transport), GO:0015979 (Photosynthesis), GO:0006139 (Nucleobase, Nucleoside, Nucleotide And Nucleic Acid Metabolic Process) and there are 14 genes uncategorized. The corresponding gene number is showed in brackets. 
Fig. 4. Cellular Component Classification

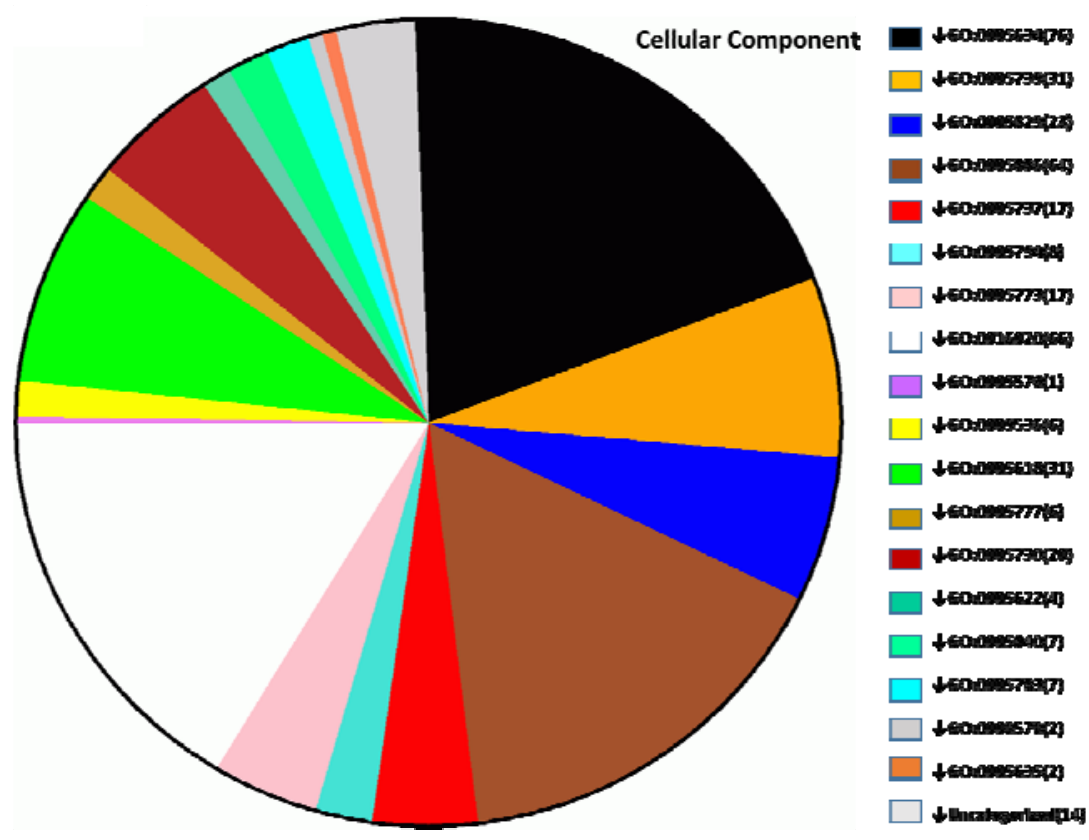

This shows the cellular component classification based on GO enrichment analysis. There are 388 genes were classified into 18 GO classes, they are GO:0005634(nucleus), GO:0005739(mitochondrion), GO:0005829(cytosol), GO:0005886(plasma membrane), GO:0005737(cytoplasm), GO:0005794(Golgi apparatus), GO:0005773(vacuole), GO:0016020(membrane), GO:0005576(extracellular region), GO:0009536(plastid), GO:0005618(cell wall), GO:0005777(peroxisome), GO:0005730(nucleolus), GO:0005622(intracellular), GO:0005840(ribosome), GO:0005783(endoplasmic reticulum), GO:0009579(thylakoid), GO:0005635(nuclear envelope) and 14 uncategorized. The corresponding gene number is showed in brackets. 


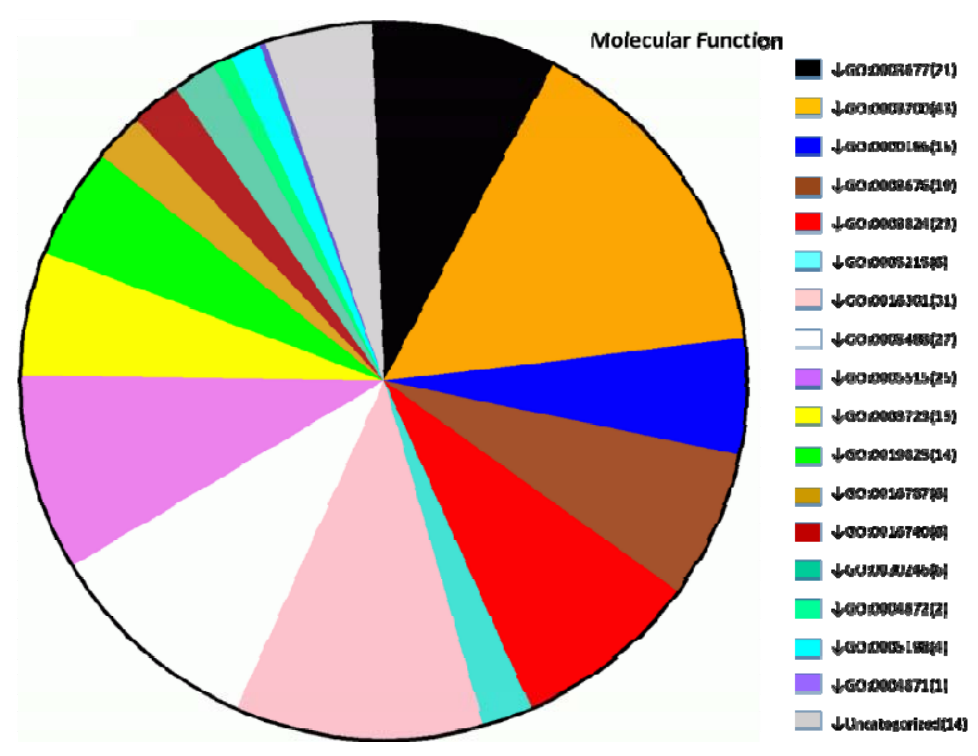

This shows the molecular function classification based on GO enrichment analysis. There are 264 genes were classified into 17 GO classes, they are GO:0003677(DNA binding), GO:0003700(sequence-specific DNA binding transcription factor activity), GO:0000166(nucleotide binding), GO:0003824(catalytic activity), GO:0005215(transporter activity), GO:0016301(kinase activity), GO:0005488(binding), GO:0005515(protein binding), GO:0003723 (RNA binding), GO:0019825(oxygen binding), GO:0016787(hydrolase activity), GO:0016740(transferase activity), GO:0030246(carbohydrate binding), GO:0004872(receptor activity), GO:0005198(structural molecule activity), GO:0004871 (signal transducer activity) and 14 uncategorized. The corresponding gene number is showed in brackets. 
Fig. 6. Gene location map

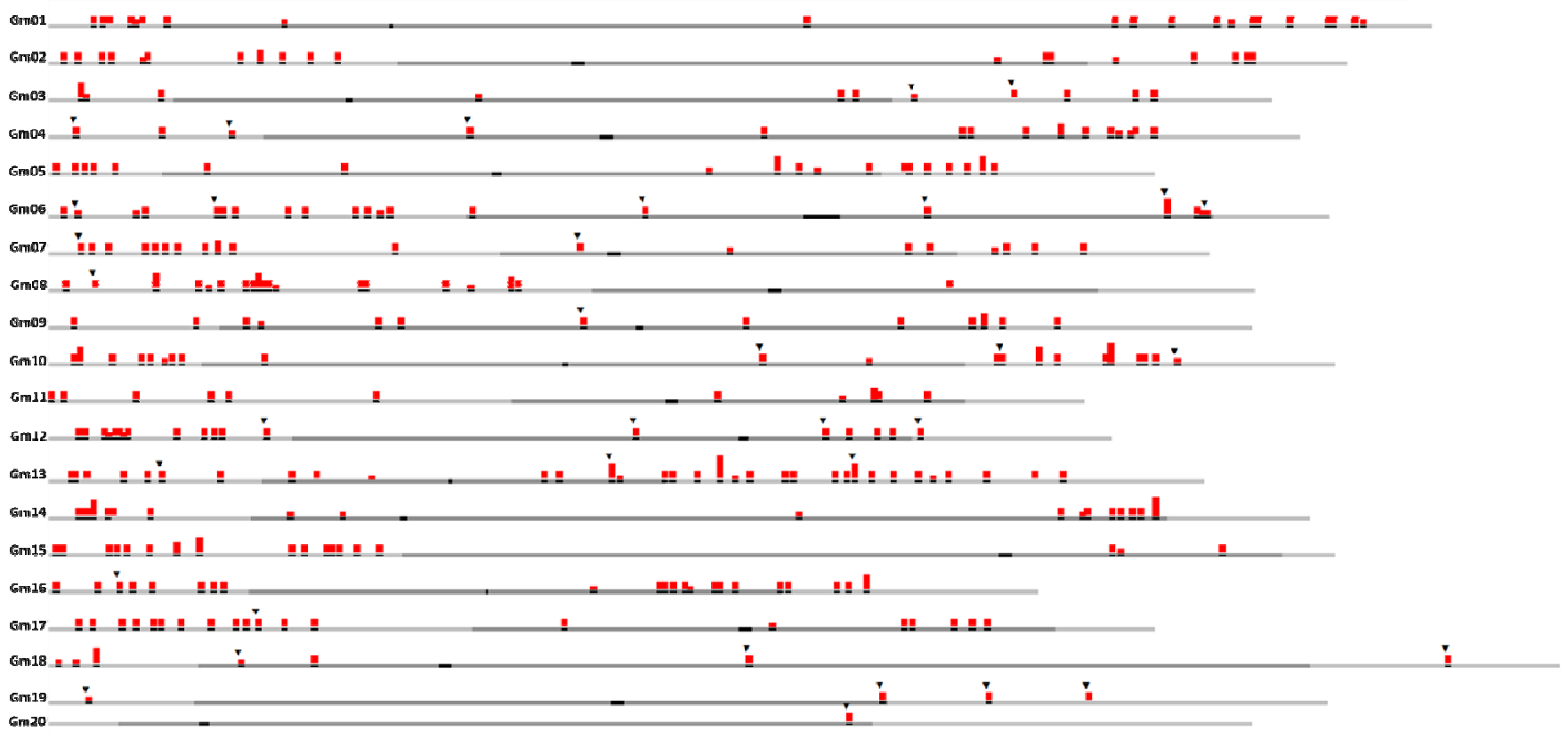

This shows the location of 328 genes, identified by our feature importance analysis. In soybase, there are 35 branching related genes were previously reported; For comparison, the 35 genes are also added to this map (marked by $\mathbf{\nabla}$ ). Color coding is used in the genome viewer to differentiate each query in a multiple FASTA submission. The height of the colored indicators is proportional to the number of BLAST hits in that genomic bin. 
Seven Additional Files:

Sup_Table1. GAPIT.MLM.Branching.GWAS.Results.csv

Sup_Table2. RF_Perm_importance.xlsx

Sup_Table3. RF_feature_score.xlsx

Sup_Table4. gene Blast result.xls

Sup_Table5. gene ontology analysis.xlsx

Sup_Table6. gene_expression information.csv

Sup_Table7. RS_HeaderT.csv 